\title{
Über die Moose,
}

die unter dem Einfluß der elektrischen Beleuchtung in das Innere

der Höhlen in Ungarn und in der Tschechoslowakei eindringen

Von A. Boros ${ }^{1}$ )

In einigen sehenswürdigen Höhlen sind großartige elektrische Beleuchtungen eingerichtet, die infolge des großen Fremdenverkehrs über einen großen Teil des Tages eingeschaltet werden. In solche Höhlen, deren Inneres ich vor einigen Jahrzehnten noch frei von grünen Pflanzen fand, dringen neuerlich Moose ein, und es kommt vor, daß einige dort auch Sporogone hervorbringen. Im folgenden berichte ich über Beobachtungen, wo ich tief in den Höhlen, in die keine Spur von Außenlicht einsickern kann, unter Einfluß der elektrischen Beleuchtung Moose konstatieren konnte.

Am Fuße der Niederen Tatra, im Tale der Dämenovaer oder Deménfalvaer „Jaskina Slobody"-Höhle (ČSR) traf ich ein wenig steriles Amblystegium varium (Hedw.) Lindb. Viel reicher erwies sich an Moosen die weltberühmte Punkva-Höhle bei Macocha (Mähren). Hier traf ich die Arten Fissidens taxifolius (L.) Hedw., Bryoërythrophyllum recursirostrum (Hedw.) Chen, Mniobryum albicans (Wahl.) Limpr., Leptobryum pyriforme (L.) Schimp., Amblystegium sarium (Hedw.) Lindb.; darunter brachte das Leptobryum reichlich gutentwickelte Sporogone. Hier ist der Farn Dryopteris filix-mas mit gutentwickeltem grünem Laub zu finden.

In Ungarn sah ich in der Höhle von Tapolca Fissidens bryoides (L.) Hedw., Gymnostomum rupestre Schleich., Tortula muralis (L.) Hedw. und Bryum capillare L.

Von den Moosen, die ich vor 30 Jahren im Eingange der Abaligeter Tropfsteinhöhle (Komitat Baranya) beobachtete, drangen Pellia Fabbroniana Raddi, Barbula cylindrica (Tayl.) Schimp. und der Farn Cystopteris filix-fragilis (L.) Borb. neuerlich in das Innere der Höhle ein. Außer diesen erhielt ich aus dem Inneren dieser Höhle noch die folgenden Moos-Arten: Weisia rutilans (Hedw.) Lindb., Bryum capillare L. var. flaccidum B. E., Eurhynchium Swartzii (Turn.) Hobk. Alle waren steril.

1) Ȧldás Utca 4, Budapest, Ungarn. 
Aus der Höhle „Anna-barlang“ bei Lillafüred neben Miskolc erhielt ich Proben, in denen ich Barbula glauca (Ryan) Podp., Taxiphyllum depressum (Bruch.) Reimers konstatieren konnte, aus der Höhle ,Szent István-barlang" ebenda bestimmte ich die folgenden Moose: Fissidens exilis Hedw., Bryoërythrophyllum recursirostrum (Hedw.) Chen, Barbula rigidula (Hedw.) Mitt., Amblystegium serpens (L.) B. E. Keine dieser bringen Sporogone.

\section{ZUSAMMENFASSUNG}

Die Einführung von elektrischer Beleuchtung in den verschiedenen Höhlen ermöglichte es einigen Moosen und Farnkräutern, in die Tiefe dieser Höhlen einzudringen, da diese früher (das heißt vor Einführung der elektrischen Beleuchtung) steril von diesen Pflanzen waren. Untersuchungen in vier Höhlen in der Tschechoslowakei und in Ungarn zeigten das Vorhandensein von Moosen, welche tief im Innern dieser Höhlen gediehen, der elektrischen Beleuchtung zufolge.

\section{ABSTRACT}

The introduction of electrical illumination into different caves makes the intrusion of some mosses and ferns into such depths of the caves possible which at previous occasions (i.e. before the installation of electrical light) were found sterile of these plants. Investigations of two caves in Czechoslovakia and 4 caves in Hungary revealed the presence of mosses thriving deep inside of these caves making use of the artificial illumination. 